\title{
DOUBLE PION PHOTOPRODUCTION IN NUCLEI
}

\author{
J.A. Gómez Tejedor, M. J. Vicente-Vacas and E.Oset \\ Departamento de Física Teórica and IFIC, \\ Centro Mixto Universidad de Valencia-CSIC \\ 46100 Burjassot (Valencia), Spain
}

\begin{abstract}
The inclusive $A\left(\gamma, \pi^{+} \pi^{-}\right) X$ reaction is studied theoretically. A sizeable enhancement of the cross section is found, in comparison with the scaling of the deuteron cross section $\left(\sigma_{d} A / 2\right)$. This enhancement is due to the modifications in the nuclear medium of the $\gamma N \rightarrow \pi \pi N$ amplitude and the pion dispersion relation. The enhancement is found to be bigger than the one already observed in the $(\pi, \pi \pi)$ reaction in nuclei.
\end{abstract}




\section{Introduction}

Because of the strong interaction of the pions with the nuclear medium, important renormalization effects are expected to appear in the pion production reactions on nuclei. In the last years the $(\pi, \pi \pi)$ process has been much studied, both theoretically [1, 2, 3] and experimentally [4, 5, 6, 7]. A quite strong sensitivity to medium effects has been found.

In this work we have studied these effects for the case of the $\left(\gamma, \pi^{+} \pi^{-}\right)$ reaction. This reaction, induced by photons, presents some advantages over the $(\pi, \pi \pi)$ case. The main one is that $\left(\gamma, \pi^{+} \pi^{-}\right)$is less peripheral and therefore it explores regions of a higher baryon density.

The $\left(\gamma, \pi^{+} \pi^{-}\right)$reaction on nucleons has been studied experimentally in refs. [8, 9], and more recently in ref. [10]. On the theoretical side, there is an early work [11] which describes the reaction using a very simple model, and the recent and much more elaborated work of ref. [12]. This latter model will be used here. As yet there are no data for this reaction in nuclei.

\section{Model for the $\mathbf{N}\left(\gamma, \pi^{+} \pi^{-}\right) \mathbf{N}$ reaction}

The model for the $p\left(\gamma, \pi^{+} \pi^{-}\right) p$ reaction developed in [12] contains the mechanisms associated to the diagrams of fig. 1, and those obtained from them by changing the time ordering. The intermediate baryon states (continuous, straight lines) include $\mathrm{N}, \mathrm{N}^{*}(1440), \mathrm{N}^{*}(1520)$ and $\Delta(1232)$. Higher resonances are expected to contribute little at the energies considered there $\left(E_{\gamma}<1000\right.$ $\mathrm{MeV})$. In this work we will study the reaction at lower energies $\left(E_{\gamma}<600\right.$ $\mathrm{MeV}$ ). Only a few of the diagrams included in [12 are important in this energy regime. They are shown in fig. 2 .

For the $n\left(\gamma, \pi^{+} \pi^{-}\right) n$ amplitude we use the same model, changing of course the corresponding isospin factors, as for the $p\left(\gamma, \pi^{+} \pi^{-}\right) p$ case, which was studied in [12]. We have checked that it reproduces quite well the cross section for $E_{\gamma}<600 \mathrm{MeV}$, also for the neutron case.

\section{$3 \quad$ Reaction in the nuclear medium}

Inside the nucleus the amplitudes will be modified. Apart from the trivial modifications to account for the Fermi motion and the Pauli blocking, vertices and propagators will change. Based on the calculations of [13 we expect very small corrections of the $\pi N N$ vertex, which we ignore. We also ignore other possible vertex corrections which we expect to be small.

The $\Delta$ propagator is strongly modified in the nucleus, and it should be dealt with carefully because the diagrams with $\Delta$ resonances provide the largest 
contribution. We take

$$
G_{\Delta}(\sqrt{s})=\frac{1}{\sqrt{s}-m_{\Delta}+i \tilde{\Gamma}_{\Delta} / 2-i \operatorname{Im} \Sigma_{\Delta}}
$$

where $\tilde{\Gamma}_{\Delta}$ is the $\Delta$ width, corrected by Pauli blocking. We take $\operatorname{Im} \Sigma_{\Delta}$ from [14], where pion quasielastic corrections, and pion two- and three-body absorption contributions to the $\Delta$ width are included. $\operatorname{Im} \Sigma_{\Delta}$ was calculated in 14] for $\sqrt{s}>1150 \mathrm{MeV}$. Below that energy we have assumed $\operatorname{Im} \Sigma_{\Delta}$ to be constant, which is consistent with the trend observed in the empirical analysis of [15], that shows a quite constant value of $\operatorname{Im} \Sigma_{\Delta}$ in a wide energy range. Furthermore for these low energies the real part of the denominator is quite large and the results are not very sensitive to $\operatorname{Im} \Sigma_{\Delta}$. The contribution of diagrams with $N$ and $N^{*}$ is much smaller, and we have not considered the medium effects in their propagators.

The meson propagators can be strongly renormalized because of the strong $\pi N$ interaction, together with the light pion mass. The pion propagator attached to a baryon line is modified in the following way (fig. 3):

$$
\begin{gathered}
D_{0}(q) \longrightarrow D_{0}(q)+D_{0}(q) U(q) V_{l}(q)+D_{0}(q) U(q) V_{l}(q) U(q) V_{l}(q)+\ldots \\
=D_{0}(q) \frac{1}{1-U(q) V_{l}(q)}
\end{gathered}
$$

where $U(q)$ is the Lindhard function for both $p h$ and $\Delta h$ excitation. Explicit expressions are found in refs. [16, 17]. To be consistent with the rest of our calculation, we use the delta width and self energy of ref. [14 in the $\Delta$-Lindhard function expressions. The longitudinal part of the spin-isospin nuclear interaction $V_{l}(q)$ is given by

$$
V_{l}(q)=F^{2}(q) \frac{f^{2}}{\mu^{2}}\left(\frac{\vec{q}^{2}}{q^{02}-\vec{q}^{2}-\mu^{2}}+g^{\prime}\right)
$$

with $g^{\prime}$ the spin-isospin correlation parameter $\left(g^{\prime}=0.6\right), \mu$ is the pion mass and $f$ is the $\pi N N$ coupling constant $\left(f^{2} / 4 \pi=0.08\right)$. The form factor is taken to be

$$
F(q)=\frac{\Lambda^{2}-\mu^{2}}{\Lambda^{2}-q^{2}}
$$

with $\Lambda=1.2 \mathrm{GeV}$.

Furthermore, we have

$$
D_{0}(q) \frac{1}{1-U(q) V_{l}(q)}=
$$




$$
\frac{1}{\left[1-\left(f^{2} / \mu^{2}\right) U(q) F^{2}(q) g^{\prime}\right]\left(D_{0}^{-1}-\Pi^{(p)}\right)}
$$

where

$$
\Pi^{(p)}=\frac{\left(f^{2} / \mu^{2}\right) \vec{q}^{2} U\left(q^{0}, \vec{q}\right) F^{2}(q)}{1-\left(f^{2} / \mu^{2}\right) g^{\prime} U\left(q^{0}, \vec{q}\right) F^{2}(q)}
$$

is the p-wave pion self-energy [18]. We include the s-wave pion self-energy substituting $\Pi^{(p)}$ by $\Pi=\Pi^{(p)}+\Pi^{(s)}$, where

$$
\Pi^{(s)}=-4 \pi\left[(1+\epsilon) b_{0} \rho+B_{0}(1+\epsilon / 2) \rho^{2}\right]
$$

with $\epsilon=\mu / m, m$ the nucleon mass, $\rho$ the nuclear density, $b_{0}=-0.008 \mu^{-1}$, $B_{0}=(-0.124+i 0.046) \mu^{-4}$ [19], slightly modified from the one of ref. [20] to account for different neutron and proton radii.

\section{$4 \gamma A \longrightarrow \pi^{+} \pi^{-} X$ amplitude in a nucleus}

We evaluate the cross section for a Fermi sea of nucleons and apply the results to finite nuclei by making use of the local density approximation. Then, following ref. [2] we get, for the cross section,

$$
\sigma=-\frac{1}{k} \int d^{3} r \operatorname{Im} \Pi(k, \rho(\vec{r}))
$$

where $\operatorname{Im} \Pi(k, \rho(\vec{r}))$ is the photon selfenergy in infinite nuclear matter corresponding to the diagram of fig. 4. The integration extends over the volume of the nucleus and $\operatorname{Im} \Pi(k, \rho(\vec{r}))$ is evaluated for the local density of the nucleus at the integration point. Only the cut shown in the figure, that corresponds to two pions in the final state, is included in the calculation. Thus,

$$
\begin{gathered}
-i \Pi(k)=-\int \frac{d^{4} p}{(2 \pi)^{4}} \int \frac{d^{4} q_{1}}{(2 \pi)^{4}} \int \frac{d^{4} q_{2}}{(2 \pi)^{4}} \sum_{\alpha} \sum_{s_{f}} \sum_{s_{i}} \overline{\sum_{p o l}}(-i) T_{\alpha}(-i) T_{\alpha}^{*} \\
\frac{i n_{\alpha}(\vec{p})}{p^{0}-E(\vec{p})-i \eta} \frac{i\left[1-n_{\alpha}\left(\vec{k}+\vec{p}-\vec{q}_{1}-\vec{q}_{2}\right)\right]}{k^{0}+p^{0}-q_{1}^{0}-q_{2}^{0}-E\left(\vec{k}+\vec{p}-\vec{q}_{1}-\vec{q}_{2}\right)+i \eta} \\
\frac{i}{q_{1}^{02}-\vec{q}_{1}^{2}-\mu^{2}+i \eta} \frac{i}{q_{2}^{02}-\vec{q}_{2}^{2}-\mu^{2}+i \eta}
\end{gathered}
$$

where $s_{i}, s_{f}$ are the initial and final nucleon spin, $\alpha$ stands for proton or neutron, and $\bar{\sum}_{p o l}$ accounts for the average of photon polarizations, $n_{\alpha}(\vec{p})$ is the occupation number for the local fermi sea, and $E(\vec{p})=\sqrt{m^{2}+\vec{p}^{2}}$. Performing the energy integrations, and including $\operatorname{Im} \Pi(k)$ in eq. 8 we get 


$$
\begin{aligned}
\sigma= & \frac{\pi}{k} \int d^{3} r \int \frac{d^{3} p}{(2 \pi)^{3}} \int \frac{d^{3} q_{1}}{(2 \pi)^{3}} \int \frac{d^{3} q_{2}}{(2 \pi)^{3}} \sum_{\alpha} \sum_{s_{f}, s_{i}} \overline{\sum_{p o l}}\left|T_{\alpha}\right|^{2} n_{\alpha}(\vec{p})\left[1-n_{\alpha}\left(\vec{k}+\vec{p}-\vec{q}_{1}-\vec{q}_{2}\right)\right] \\
& \frac{1}{2 \omega\left(\vec{q}_{1}\right)} \frac{1}{2 \omega\left(\vec{q}_{2}\right)} \delta\left(k^{0}+E(\vec{p})-\omega\left(\vec{q}_{1}\right)-\omega\left(\vec{q}_{2}\right)-E\left(\vec{k}+\vec{p}-\vec{q}_{1}-\vec{q}_{2}\right)\right)
\end{aligned}
$$

This equation, applied to the nucleon alone, i.e., by taking $2 \int d^{3} r \int \frac{d^{3} p}{(2 \pi)^{3}} n_{\alpha}(\vec{p})=$ 1 , and neglecting Pauli blocking, gives the ordinary formula for the $\gamma N \longrightarrow$ $\pi^{+} \pi^{-} N$ cross section.

Moreover, we must consider the distortion of the outgoing pion waves, and of the incoming photon. For the incoming photon due to its small interaction with nuclei we neglect the distortion effects. For the outgoing pions our calculation of the distortion takes into account the reduction of pion flux due to absorption. This is done by multiplying the integrand of eq. 10 by a function that accounts for two- and three-body absorption. See section 3 of ref. [2] for the explicit formula of this function.

\section{Effect of the binding of the pions in the nuclear medium}

One key point of this reaction is the phase space for the $\vec{p}, \overrightarrow{q_{1}}, \overrightarrow{q_{2}}$ integration governed by the energy conservation shown explicitly by the $\delta$ function of eq. 10. To be rigorous we also must include in eq. 10 the potential energies affecting the pions and nucleons inside the nucleus. For the nucleons any such potential, that does not depend too strongly on the energy, will modify very little the balance of energies, as it will appear with a different sign for the initial and the final nucleon. However, for the pions there will be no cancellation. At the energies we have selected, the pion self-energy will be attractive, except for very low energy pions, where the repulsive s-wave interaction dominates. This means that the phase space, at a local level, will now be increased, as compared to the reaction on a nucleon, because for a certain value of the pion momentum, $\vec{q}$, the energy in the medium, $\tilde{\omega}(\vec{q})$, will be smaller. Hence, one can accommodate larger values of $\vec{q}_{1}$ and $\vec{q}_{2}$ in the integration

As mentioned in the introduction, the photon is very weakly distorted and enters deep inside the nucleus, producing a less peripheral reaction than the

\footnotetext{
${ }^{1}$ This procedure of renormalizing the pions in pion production processes, in connection with the local density approximation, has been shown to be formally equivalent to the use of pion wave functions in finite nuclei and the subsequent evaluation of nuclear matrix elements under certain semiclassical approximations and is numerically very accurate. 21]
} 
$(\pi, \pi \pi)$ one studied in 2]. Therefore, we expect a bigger change of the total cross section due to the binding of the pions than in the $(\pi, \pi \pi)$ case, where factors of two or three are reached.

In order to account for this effect we follow the same procedure as in [2]. Before making the energy integration in eq. 9, we substitute the free pion propagator $D_{0}(q)$ by the pion propagator in the medium $D(q)$ given by

$$
D(q)=\frac{1}{q^{02}-\vec{q}^{2}-\mu^{2}-\Pi\left(q^{0}, \vec{q}\right)}
$$

where the pion self-energy $\Pi=\Pi^{(p)}+\Pi^{(s)}$, is the sum of a p-wave and an s-wave part, eq. 6, 7.

The modifications to eq. 10 are simple. In first place, the $q_{1}^{0}, q_{2}^{0}$ integrations, instead of $(2 \omega(\vec{q}))^{-1}$, give now

$$
\operatorname{Res} D(q)=\left.\frac{1}{2 q^{0}-\partial \Pi / \partial q^{0}}\right|_{q^{0}=\tilde{\omega}(\vec{q})}
$$

where $\tilde{\omega}(\vec{q})$ is the energy of a pion with momentum $\vec{q}$ inside the nuclear medium, given by the poles of the propagator $D(q)$, obtained from the equation

$$
\tilde{\omega}(\vec{q})^{2}-\vec{q}^{2}-\mu^{2}-\operatorname{Re} \Pi(\tilde{\omega}(\vec{q}), \vec{q})=0
$$

Here, we suppose that $I m \Pi$ is small, and thus it is neglected.

In addition, in the argument of the $\delta$ function of eq. 10, $\omega(\vec{q})$ will be substituted by $\tilde{\omega}(\vec{q})$.

\section{$6 \quad$ Results and Discussions}

We have evaluated the cross section of the reaction $\left(\gamma, \pi^{+} \pi^{-}\right)$for ${ }^{12} \mathrm{C}$ and ${ }^{40} \mathrm{Ca}$. The results are shown in figs. 5 and 6 . In both figures, the curve labelled 1 corresponds to a simple impulse approximation where the free $\gamma N \rightarrow \pi \pi N$ amplitude has been used. The only nuclear medium effects included in this curve are the Fermi motion of the initial nucleon and the absorption of the final pions. The curve labelled 2 is the scaling of the deuteron cross section. Actually, we use $\left(Z \sigma_{p}+(A-Z) \sigma_{n}\right) / A$, with $\sigma_{p}$ and $\sigma_{n}$ the experimental values of the cross sections for proton and neutron respectively. At high energies, the dominant nuclear effect in the impulse approximation of curve 1 is pion absorption which reduces the cross section. Of course, absorption is more important in the case of ${ }^{40} \mathrm{Ca}$ where the results of curve 1 are four times smaller than the scaling of curve 2. For ${ }^{12} C$ this factor is almost three. At low energies, however, we can appreciate the effect of the shift of the threshold produced by the Fermi motion of the nucleons. Because of this, curve 1 is bigger than the scaling at very low energies. 
The upper curve (labelled 3) corresponds to the full model as described before. The results are larger than the scaling of curve 2. The enhancement is due to the binding of the final pions, and the changes of the amplitude. Both effects are approximately of the same importance. At high energies the enhancement is less spectacular partly because of the absorption, which becomes stronger, and also the binding is less effective.

We have also added in figs. 5 and 6 another curve, labelled 4, which corresponds to neglecting the renormalization of the two pion propagators of eq. 9, but keeping the renormalization of the internal delta and pion lines in the amplitudes according to eqs. 11 and 2 respectively. This is equivalent to neglecting the renormalization of the outgoing pions. As we see in the curves labelled 4 in figs. 5 and 6 , the renormalization of these internal propagators alone leads to a small reduction of the cross section at photon energies around 500-600 MeV. One should, however, bear in mind that the effect of this "internal" renormalization is different when simultaneously the "external" pions are renormalized. This is so because the external pion renormalization, which changes the pion dispersion relation, makes the internal pion closer to the on shell situation as the density increases, leading to some enhancement of the amplitudes which helps somewhat in the enhanced cross section found in figs. 5 and 6.

Although the enhancement produced by the quoted medium effects is approximately the same for ${ }^{40} \mathrm{Ca}$ and ${ }^{12} \mathrm{C}$, absorption is much stronger for the ${ }^{40} \mathrm{Ca}$ case. Thus $\sigma / A$ decreases when we pass from light to medium or heavy nuclei. So far, there are not experimental data for the $\left(\gamma, \pi^{+} \pi^{-}\right)$reaction in nuclei. However, there are results for the total photonuclear cross section [22]. Bellow $500 \mathrm{MeV}$ the two pions cross section is very small compared to the total one. However we found that around $600 \mathrm{MeV}$ the $\left(\gamma, \pi^{+} \pi^{-}\right)$cross section is a sizeable part of the total (approximately one half).

We have studied the stability of these results when the spin-isospin parameter $g^{\prime}$ is slightly modified, and we have found that changing $g^{\prime}$ from 0.5 to 0.7 the results change about a $10 \%$, being larger for small $g^{\prime}$.

In fig. 7 we compare the energy spectra of the negative pions that would be produced with the simple model of curve 1 (dashed line) and with the full model (continuous line). The results are similar for the positive pions. We have to remark that this figure can not be compared directly to the experiment because the spectra correspond to the moment of production and possible quasielastic scatterings of the pions have not been considered in the calculations. Note that this kind of final state interactions would not modify the total cross section, but would certainly change the energy and angular differential cross sections. We can observe in the figure that the enhancement affects mostly the pions of energies around $220 \mathrm{MeV}$ and much less the low and high energy pions. This clearly marks the regions of pion energies where 
the medium effects are more relevant.

We have also analyzed how peripheral is the reaction for several nuclei and at several energies. We have found that most of the pion production takes place at densities around half of the central nuclear density, and it is a bit more peripheral at low energies.

In summary, we have studied the inclusive $\left(\gamma, \pi^{+} \pi^{-}\right)$reaction in nuclei for ${ }^{12} \mathrm{C}$ and ${ }^{40} \mathrm{Ca}$. We have found important nuclear effects for this reaction. The enhancement of the cross section is larger that in the much studied $(\pi, \pi \pi)$ reaction, as it was expected due to the less peripheral character of this reaction. Experiments to test this large and interesting renormalization effect would be most welcome.

\section{Acknowledgements}

This work was partially supported by CICYT, contract AEN 93-1205. One of us, J.A. Gómez Tejedor, wishes to acknowledge financial support from the Institució Valenciana d'Estudis i Investigació. 


\section{References}

[1] J.M. Eisenberg, Phys. Lett. 93B (1980) 12; J. Cohen and J.M. Eisenberg, Nucl. Phys. A395 (1983) 389; J. Cohen, J. of Phys. G9 (1983) 621

[2] E. Oset and M.J. Vicente Vacas, Nucl. Phys. A446 (1985) 584; Nucl. Phys. A454 (1986) 637

[3] P. Schuck, W. Nöremberg and G. Chanfray, Z. Phys. A330 (1988) 119

[4] N. Grion et al., Nucl. Phys. A492 (1989) 509

[5] R. Rui et al., Nucl. Phys. A517 (1990) 455

[6] A. Rahav et al., Phys. Rev. Lett. 66 (1991) 1279

[7] D. Vetterli et al., Nucl. Phys. A548 (1992) 541

[8] Aachen-Berlin-Bonn-Hamburg-Heidelberg-München collaboration, Phys. Rev. 175 (1968) 1669

[9] G. Gialanella et al., Nuovo Cimento LXIII A (1969) 892

[10] G. Audit et al., INFN-BE-93-01, preprint.

[11] L. Lüke and P. Söding, Springer Tracts in Modern Physics 59 (1971) 39

[12] J.A. Gómez Tejedor and E. Oset, Nucl. Phys. A571 (1994) 667

[13] E. Oset and W. Weise, Phys. Lett. 60B (1976) 141

[14] E. Oset, L.L. Salcedo and D. Strottman, Phys. Lett. 165B (1985)13;

E. Oset and L.L. Salcedo, Nucl. Phys. A468(1987) 631

[15] Y. Horikawa, M. Thies and F. Lenz, Nucl. Phys. A345 (1980)386

[16] A.L. Fetter and J.D. Walecka, Quantum Theory of Many Particle Systems, (McGraw Hill, NY, 1971)

[17] E. Oset and A. Palanques-Mestre, Nucl. Phys. A359 (1981) 289

[18] E. Oset, H. Toki and W. Weise, Phys. Reports 83 (1982) 281

[19] C. García-Recio, J. Nieves and E. Oset, Nucl. Phys. A547 (1992) 473.

[20] O. Meirav, E. Friedman, R.R. Johnson, R. Olszewski and P. Weber, Phys. Rev. C40 (1989) 843. 
[21] E. Oset, P. Fernández de Córdoba, J. Nieves, A. Ramos and L.L. Salcedo, Prog. Theor. Phys. to be published.

[22] N. Bianchi et al., Nucl. Phys. A553 (1993) 631c. 


\section{Figure Captions}

Fig. 1 Feynman diagrams of the model of ref. [12] for the reaction $\gamma p \rightarrow$ $p \pi^{+} \pi^{-}$. Continuous straight lines: baryons. Dashed lines: pions. Wavy lines: photons and rho mesons (marked explicitly).

Fig. 2 Simplified model for the $\gamma p \rightarrow p \pi^{+} \pi^{-}$reaction. It contains all relevant diagrams for $E_{\gamma}<600 \mathrm{MeV}$.

Fig. 3 Diagrammatic representation of the renormalization of the pion pole terms in a nuclear medium.

Fig. 4 Diagram of a piece of the photon nucleus optical potential having as a source of imaginary part the cut corresponding to $p h 2 \pi$ excitation (dotted line).

Fig. 5 Cross section per nucleon for the ${ }^{12} C\left(\gamma, \pi^{+} \pi^{-}\right)$reaction. Dot-dashed line (labelled 1): Simple impulse approximation. Dashed line (labelled 2): Scaling of the deuteron cross section. Continuous line (labelled 3): Full model. Long dashed-dotted curve (labelled 4): cross section renormalizing the internal pion and delta lines in the amplitudes and ignoring the renormalization of the external pion lines.

Fig. 6 Same as Fig. 5 for the ${ }^{40} \mathrm{Ca}$.

Fig. 7 Energy spectra of the negative pions for ${ }^{40} \mathrm{Ca}$ and $E_{\gamma}=450 \mathrm{MeV}$. Continuous line: Full model. Dashed line: Simple impulse approximation. 
This figure "fig1-1.png" is available in "png" format from: http://arxiv.org/ps/nucl-th/9411029v3 
This figure "fig2-1.png" is available in "png" format from: http://arxiv.org/ps/nucl-th/9411029v3 


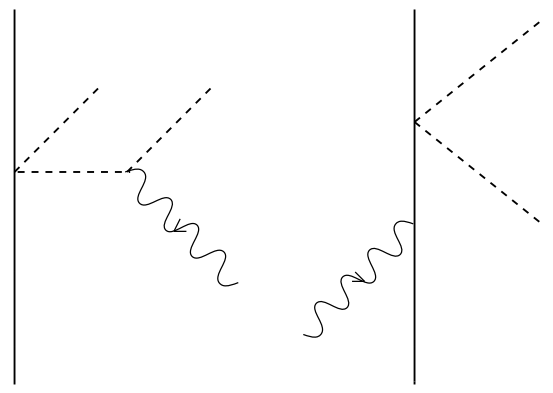

a)

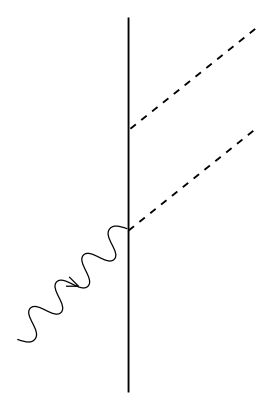

c)

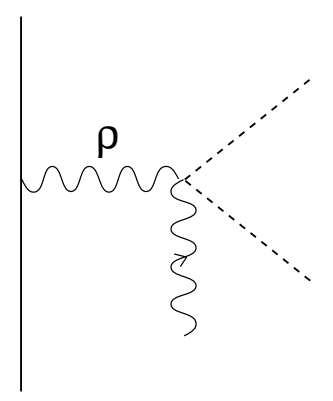

g)

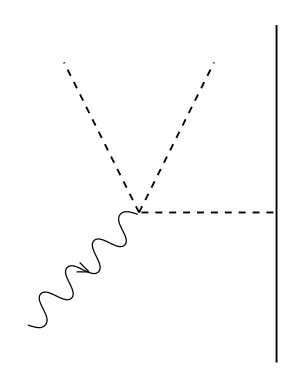

j)

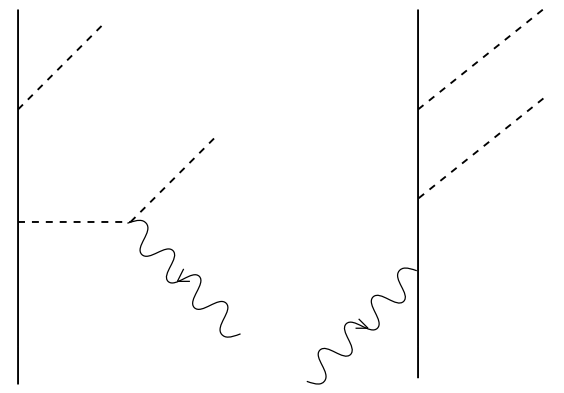

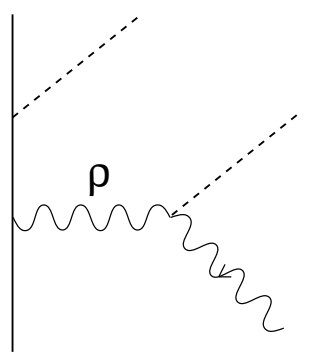

i) e)

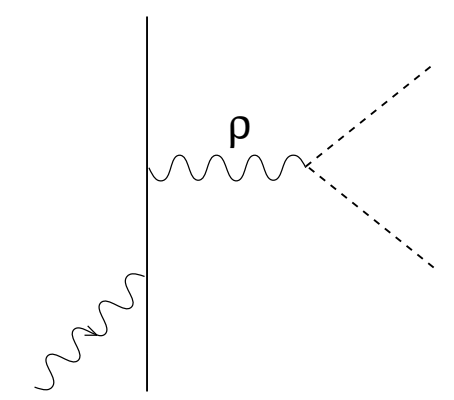

h)

d)

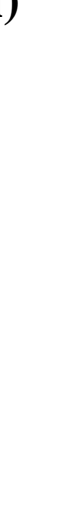

Fig. 1 
This figure "fig1-2.png" is available in "png" format from: http://arxiv.org/ps/nucl-th/9411029v3 
This figure "fig2-2.png" is available in "png" format from: http://arxiv.org/ps/nucl-th/9411029v3 


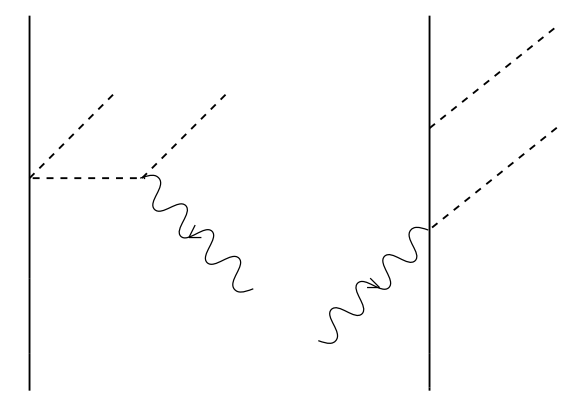

a)

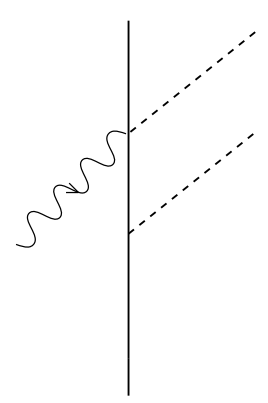

b)

c)

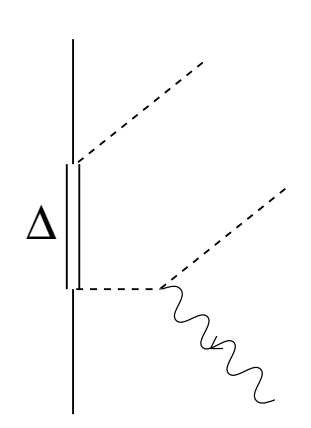

g)

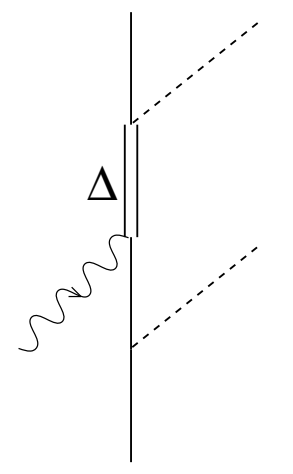

h)

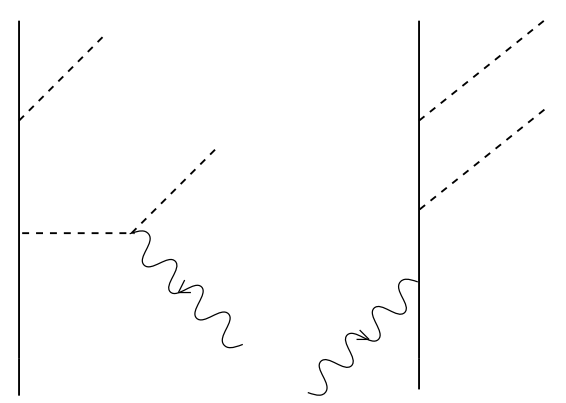

e)

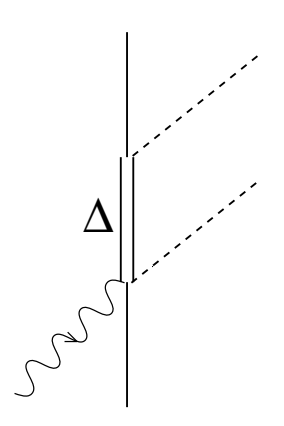

f)

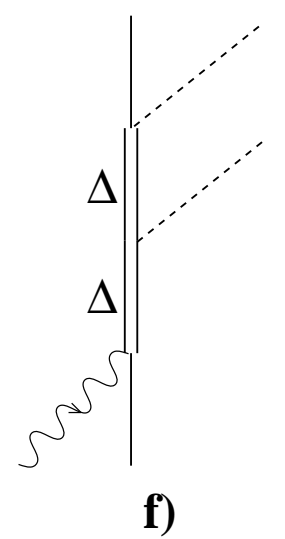

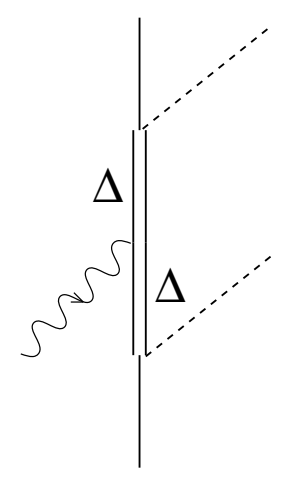

k)

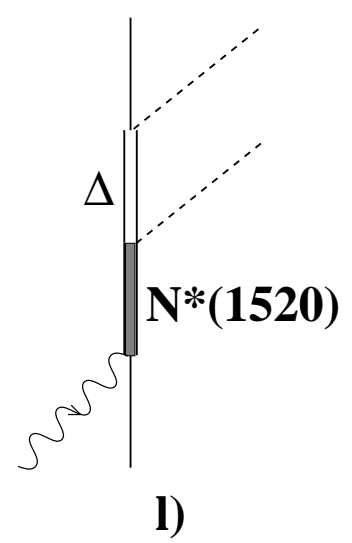

Fig. 2 
This figure "fig1-3.png" is available in "png" format from: http://arxiv.org/ps/nucl-th/9411029v3 


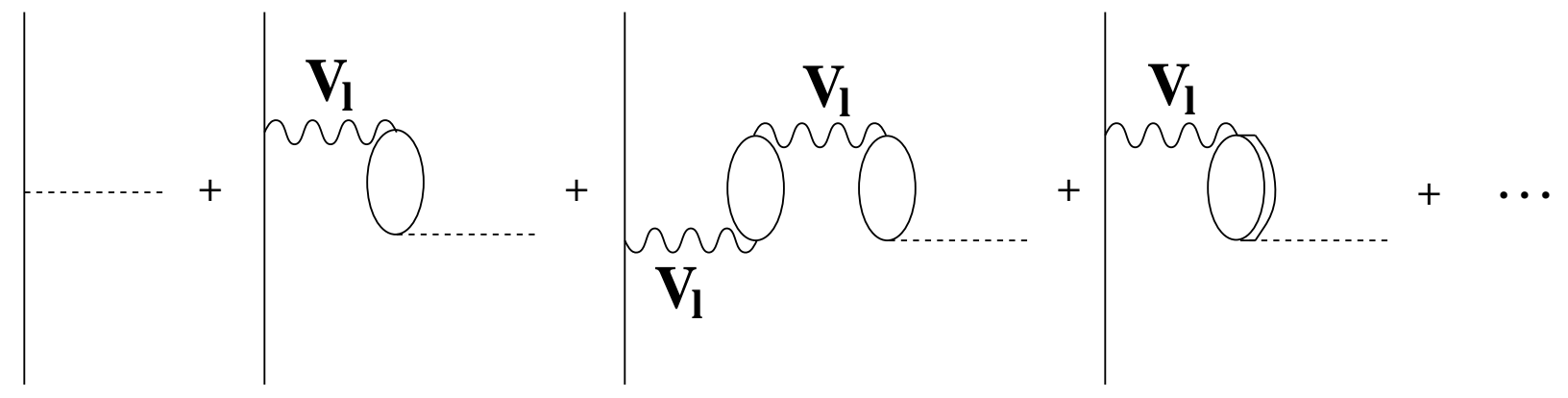

Fig. 3 
This figure "fig1-4.png" is available in "png" format from: http://arxiv.org/ps/nucl-th/9411029v3 


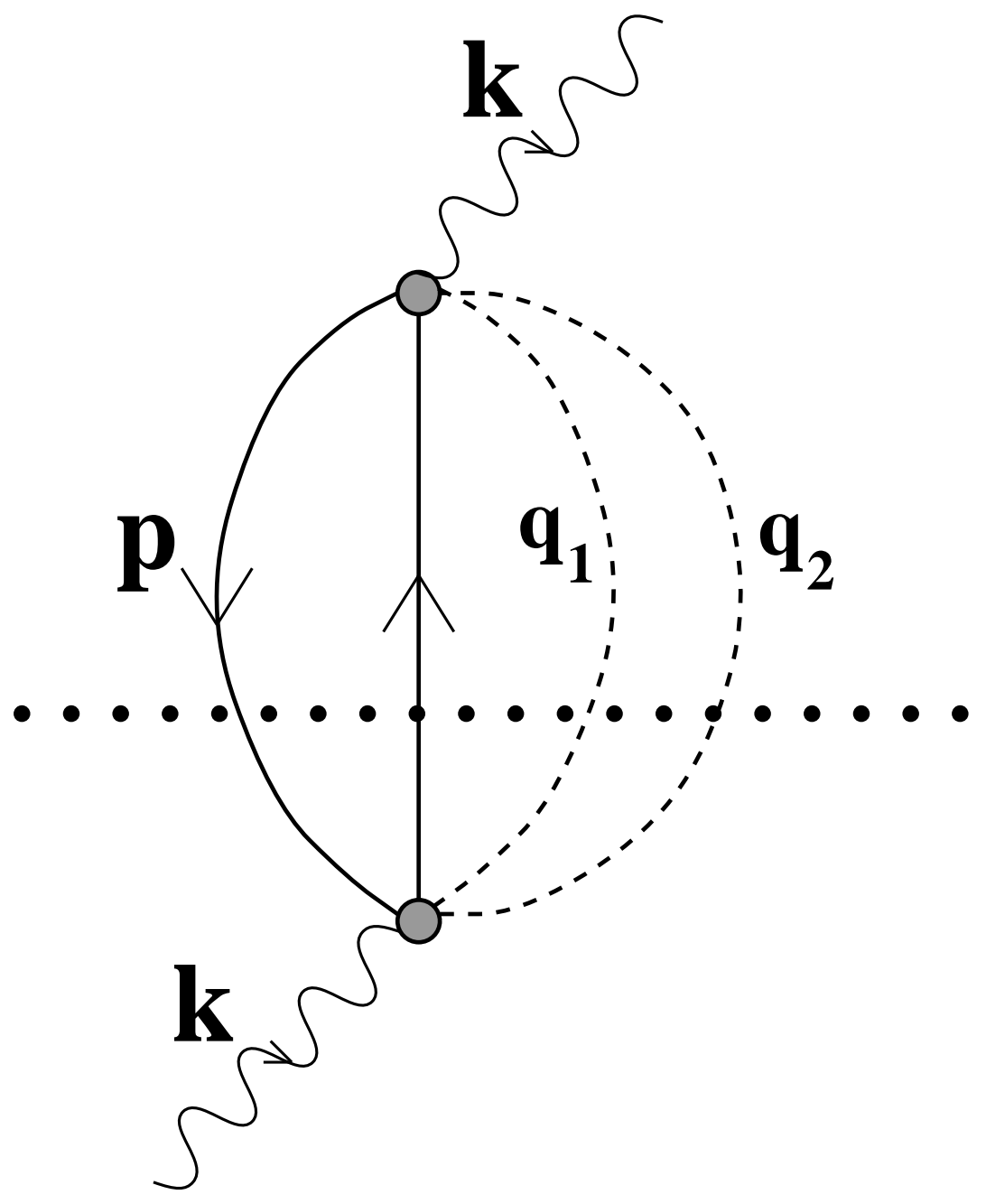

Fig. 4 
This figure "fig1-5.png" is available in "png" format from: http://arxiv.org/ps/nucl-th/9411029v3 


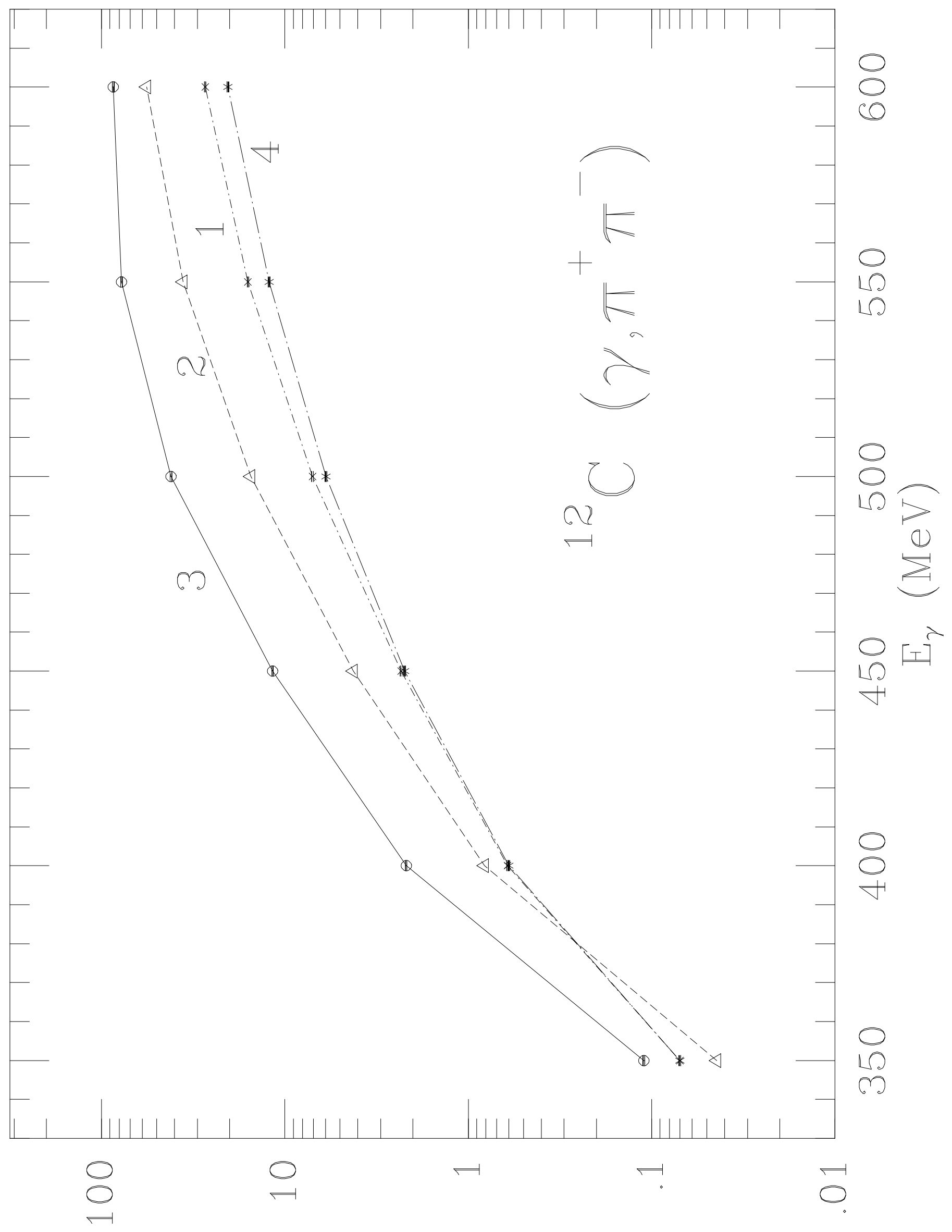

Fig. 5 


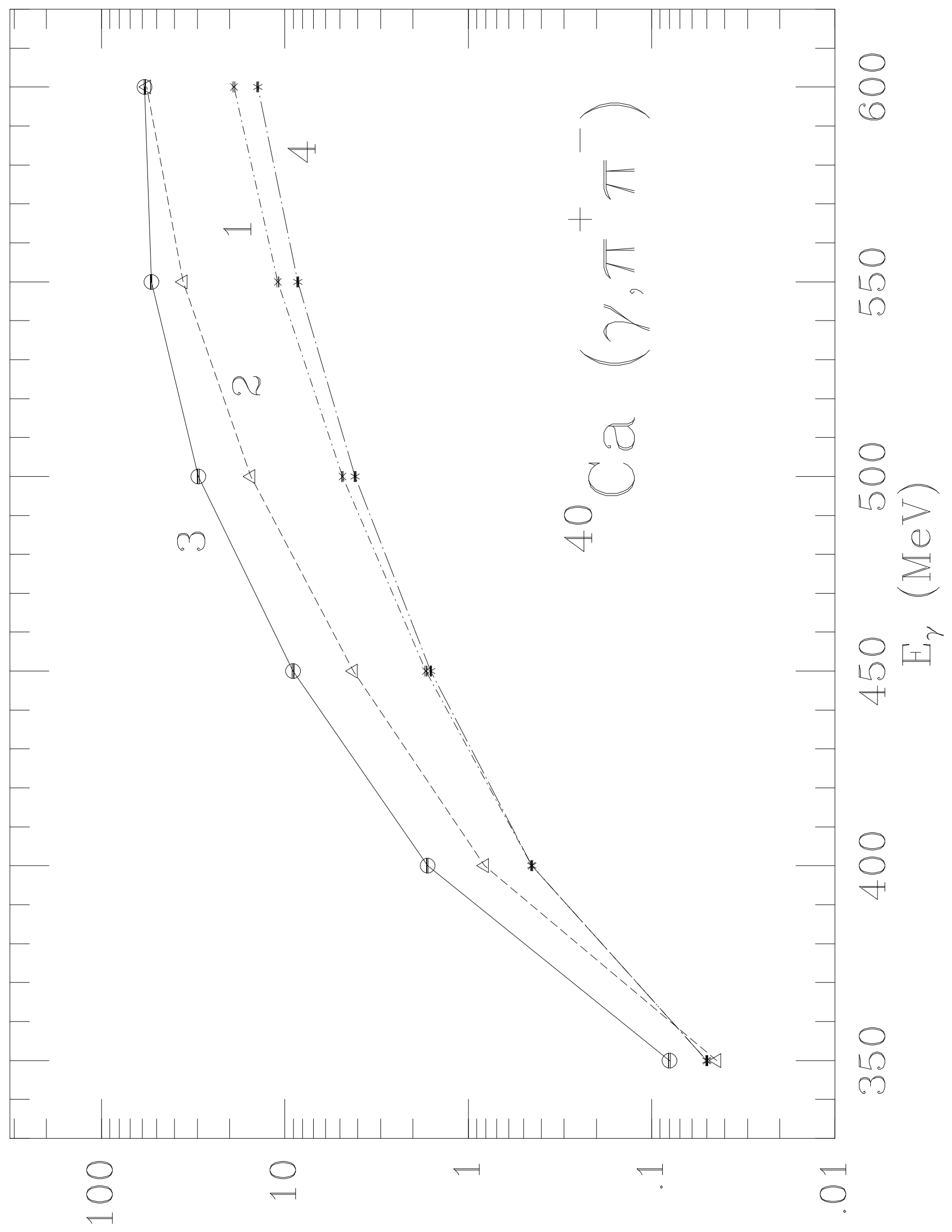

Fig. 6 


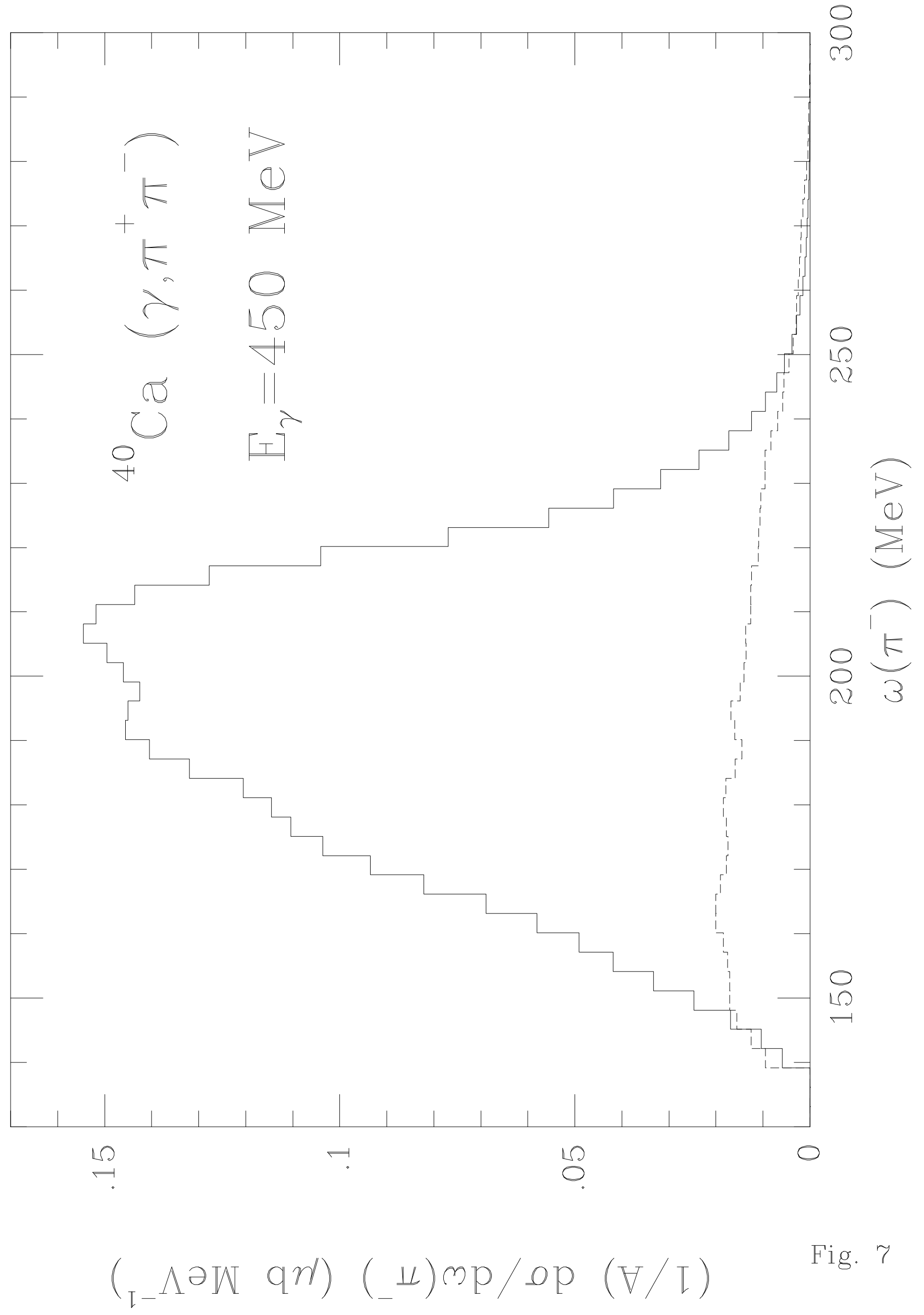

